\title{
Participação do setor público no fornecimento de medicamentos para dislipidemias em estudo de base populacional
}

\author{
Public sector participation in the supply of dyslipidemia \\ medication in a population-based study
}

Airton José Petris ${ }^{1}$
Regina Kazue Tanno de Souza ${ }^{1}$
Maira Sayuri Sakay Bortoletto ${ }^{1}$
${ }^{1}$ Departamento de Saúde Coletiva, Centro de Ciências da Saúde, Universidade Estadual de Londrina. Av. Robert Kock 70, Vila Operária. 86039-440

Londrina PR Brasil. airton.petris@gmail.com
Abstract The use of medications for the treatment of dyslipidemia is relevant in the control of cardiovascular disease. This article aims to analyze the prevalence, the use and the participation of the public sector in the supply of medication for adults aged 40 years and above using pharmacotherapy for dyslipidemia control living in a city in the southern region of Brazil. A cross-sectional, population-based study was conducted. Household interviews were staged with 1180 individuals aged over 40 living in Cambé, State of Paraná, of which 967 took laboratory examinations. The prevalence of dyslipidemia was $69.2 \%$, of which $16.1 \%$ were taking medication. Among individuals undergoing treatment for dyslipidemia, 22.2\% had adequate test results. Lipid-lowering medication used were simvastatin (81.5\%) and bezafibrate (6.5\%), mainly obtained by direct payment to private pharmacies and drug stores (52.2\%) and NHS services (33.6\%). A high prevalence of dyslipidemias was observed in population terms, together with a low level of dyslipidemia control and low participation of the public sector regarding the supply of medication compared to acquisition through direct payment for medication in private pharmacies. These results suggest a limited range of public policy for control of dyslipidemia. Key words Dyslipidemia, Prevalence, Pharmaceutical services
Resumo A utilização de medicamentos para tratamento das dislipidemias é relevante no controle das doenças cardiovasculares. O objetivo deste artigo é analisar a prevalência, a utilização e a participação do setor público no fornecimento de medicamentos para as pessoas a partir de 40 anos em farmacoterapia de controle das dislipidemias, residentes em um município da região Sul do Brasil. Estudo transversal de base populacional. Foram entrevistados no domicilio 1180 indivíduos a partir de 40 anos residentes em Cambé/PR, dos quais 967 realizaram exames laboratoriais. A prevalência de dislipidemias foi de 69,2\%, dos quais $16,1 \%$ utilizavam medicamentos. Entre os indivíduos em tratamento para as dislipidemias, 22,2\% apresentaram resultados de exames adequados. Os fármacos hipolipemiantes mais utilizados foram sinvastatina $(81,5 \%)$ e bezafibrato (6,5\%), obtidos principalmente por pagamento direto em farmácias e drogarias privadas (52,2\%) e serviços próprios do SUS (33,6\%). Em nível populacional a prevalência das dislipidemias foi elevada, o seu controle baixo, com menor participação do setor público no fornecimento dos medicamentos do que a aquisição mediante pagamento direto em farmácias e drogarias privadas, sugerindo alcance limitado das políticas públicas de controle das dislipidemias.

Palavras-chave Dislipidemias, Prevalência, Assistência farmacêutica 


\section{Introdução}

As doenças cardiovasculares (DCV) têm-se caracterizado como um problema de saúde pública mundial, com taxas de prevalência elevadas, sendo responsáveis por aproximadamente $30 \%$ das mortes $^{1,2}$. Apesar do aumento na ocorrência das DCV, observa-se, nos países desenvolvidos, diminuição do risco de morte a partir de $1970^{3}$. Mesmo nos países em diferentes estágios de transição demográfica e de desenvolvimento, com a incorporação de novas tecnologias, ocorreu redução da letalidade. Todavia, uma parcela importante dos casos ainda decorre do controle inadequado dos fatores de risco ${ }^{4}$.

No Brasil, o Ministério da Saúde tem formulado linhas de ações de enfrentamento dos principais fatores de risco cardiovascular por meio de intervenções globais ou por estratégias de identificação dos riscos individuais'. Para isso, são utilizados algoritmos como o Escore de Risco de Framingham, o Escore de Risco de Reynolds, o Escore de Risco Global e o Escore de Risco pelo Tempo de Vida, que permitem classificar os indivíduos em graus de risco alto, intermediário e baixo ${ }^{5}$. Em todos os algoritmos, as dislipidemias, definidas como alterações nos níveis do colesterol e suas frações e de triglicerídeos, são associadas à ocorrência de eventos cardiovasculares graves ${ }^{5}$.

$\mathrm{O}$ tratamento não medicamentoso das dislipidemias é sempre indicado, sendo que nas situações nas quais as mudanças no estilo de vida não logram êxito no seu controle são recomendadas intervenções farmacoterapêuticas. Hipolipemiantes com grande potencial de redução da hipercolesterolemia, especialmente do LDL-C, foram desenvolvidos nas últimas décadas, com destaque para o subgrupo farmacológico das estatinas, que foi incluído na lista de medicamentos do Programa de Medicamentos Excepcionais do Ministério da Saúde para controlar as dislipidemias em pessoas com alto risco cardiovascular ${ }^{6}$. Em 2006, foram incluídos nesta lista fármacos do subgrupo químico fibratos ${ }^{7}$, sendo a sinvastatina transferida para o Componente Básico da Assistência Farmacêutica em 2009. Em 2010, a sinvastatina passou a ser disponibilizada mediante ressarcimento, dentro do Programa Farmácia Popular do Brasil ${ }^{9}$.

Estudos que tratam deste tema em nível populacional são escassos e a maioria refere-se a programas assistências específicos, principalmente públicos, a partir de dados obtidos do sistema de vigilância epidemiológica. O objetivo do presente estudo foi analisar a prevalência, a utilização e o acesso à farmacoterapia de controle das dislipidemias em pessoas a partir de 40 anos, residentes em um município de médio porte.

\section{Métodos}

Estudo de base populacional, parte do trabalho mais abrangente "Doenças cardiovasculares no Estado do Paraná: mortalidade, perfil de risco, terapia medicamentosa e complicações" (PROJETO VIGICARDIO), realizado em Cambé-PR no primeiro semestre de 2011, município com 96.733 habitantes, dos quais $96,1 \%$ residentes na área urbana e $36,9 \%$ com 40 anos ou mais ${ }^{10}$.

O município conta com 12 Unidades de Saúde da Família $(U S F)^{11}$. A assistência farmacêutica é prestada pela estrutura própria do serviço municipal de saúde e por estabelecimentos privados, muitos vinculados ao Programa Aqui Tem Farmácia Popular (PAFP). A atenção no setor público é estruturada a partir da Central de Abastecimento. Os medicamentos da Assistência Farmacêutica Básica são disponibilizados na Farmácia Pública Municipal, em todas as USF e na Unidade de Pronto Atendimento 24 horas $^{11}$.

A população de estudo foi constituída por uma amostra representativa de adultos de 40 anos e mais residentes em todos os setores censitários urbanos (86). Para a definição do tamanho da amostra utilizou-se o aplicativo StatCalc do programa Epi Info 3.5.3 e foi considerada uma prevalência esperada de $50 \%$, margem de erro de $3 \%$ e intervalo de confiança de $95 \%$. A amostra final, estratificada por sexo e faixas etárias quinquenais, totalizou 1339 indivíduos com 40 anos ou mais, prevendo-se $25 \%$ de eventuais perdas e garantia de proporcionalidade em cada setor. Definiu-se como critério de exclusão a impossibilidade de responder a entrevista por parte dos indivíduos, desde que não acompanhados de um cuidador.

Os dados foram coletados no primeiro semestre de 2011 por meio de entrevistas, realizadas por alunos de graduação e pós-graduação da área de saúde da Universidade Estadual de Londrina, e da coleta de sangue para exames laboratoriais. As informações sobre os medicamentos utilizados e a identificação de seus componentes farmacológicos foram obtidas nas entrevistas e confirmadas com a apresentação dos medicamentos, embalagens ou prescrição médica. As questões sobre os subgrupos terapêuticos e os locais de obtenção de medicamentos foram adaptadas do questionário utilizado em estudo sobre o acesso dos idosos a medicamentos gratuitos do Projeto para a Expansão e Consolidação da Saúde da Família' ${ }^{12}$. 
A coleta do material biológico foi realizada por uma equipe com experiência profissional. Os exames laboratoriais foram processados no Laboratório de Análises Clínicas do Hospital Regional do Norte do Paraná em autoanalisador bioquímico (Dimension ${ }^{\varpi}$ ), com uso de kits de reagentes da marca Siemens (colesterol total, colesterol HDL, triacilglicerol e glicose). A determinação do LDL-C foi estimada pela equação de Friedwald [LDL-C = Colesterol total - (HDL-C $+\mathrm{TG} / 5)]$.

As dislipidemias primárias foram classificadas de acordo com as concentrações plasmáticas dos lipídeos Colesterol Total (CT), Lipoproteínas de baixa densidade (LDL-C), Triglicerídeos (TG) e Lipoproteínas de alta densidade (HDL-C): hipercolesterolemia isolada (elevação isolada do LDL-C para valores iguais ou superiores a 160 $\mathrm{mg} / \mathrm{dl}$ ), hipertrigliceridemia isolada (elevação isolada dos TG para valores iguais ou superiores a $150 \mathrm{mg} / \mathrm{dl}$ ), hiperlipidemia mista (com valores aumentados da fração LDL-C iguais ou superiores a $160 \mathrm{mg} / \mathrm{dl}$ e TG com valores iguais ou superiores a $150 \mathrm{mg} / \mathrm{dl}$ ) e HDL-C baixo (redução isolada do HDL-C em homens para valores menores do que $40 \mathrm{mg} / \mathrm{dl}$ e em mulheres para valores menores que $50 \mathrm{mg} / \mathrm{dl}$ ou em associação com aumento de LDL-C ou de $\mathrm{TG}^{5}$.

Os indivíduos que apresentaram resultados de exames laboratoriais com LDL-C com valores $\geq 160 \mathrm{mg} / \mathrm{dl}$ e/ou triglicérides com valores $\geq$ $150 \mathrm{mg} / \mathrm{dl}$ e/ou HDL-C baixos - em mulheres < $50 \mathrm{mg} / \mathrm{dl}$ e em homens $<40 \mathrm{mg} / \mathrm{dl}-$ e os que, embora tivessem as frações lipídicas dentro dos parâmetros desejáveis, faziam uso de hipolipemiantes e apresentaram as embalagens correspondentes, foram classificados na categoria com dislipidemia. Os grupos terapêuticos considerados foram estatinas, inibidores da absorção do colesterol e fibrato. Os fármacos identificados foram classificados segundo seus subgrupos químicos, farmacológicos e terapêuticos, de acordo com a Classificação ATC (The anatomical therapeutic chemical classification system), referida pela WHO como a unidade de medida padrão-ouro em pesquisas internacionais sobre a utilização de drogas $^{13}$. Entre os que utilizavam medicamentos hipolipemiantes foram considerados controlados aqueles com LDL-C com valores $<160 \mathrm{mg} / \mathrm{dl}$ e/ ou triglicérides com valores $<150 \mathrm{mg} / \mathrm{dl}$ e/ou HDL-C elevados - em mulheres $>50 \mathrm{mg} / \mathrm{dl} \mathrm{e} \mathrm{em}$ homens $>40 \mathrm{mg} / \mathrm{dl}^{5}$.

Para o processamento das informações foram criados três bancos de dados. Um de caracterização dos indivíduos, outro com todos os medicamentos utilizados e um terceiro horizontali- zado a partir dos dados do primeiro com os do segundo banco. Para inclusão no banco de dados horizontalizado os nomes dos medicamentos, comerciais ou genéricos, foram revisados e/ou transformados para a Denominação Comum Brasileira (DCB), utilizando-se os bulários eletrônicos disponibilizados pelos fabricantes.

Para análise dos resultados foi utilizado o programa SPSS (Statistical Package for the Social Sciences for Windows), versão 19. As diferenças entre as proporções foram verificadas pelo teste do Qui-quadrado de Pearson ou pelo Teste Exato de Fisher, considerando-se estatisticamente significativo o valor de $\mathrm{p}<0,05$.

O projeto foi aprovado pelo Comitê de Ética em Pesquisa Envolvendo Seres Humanos da Universidade Estadual de Londrina. As entrevistas foram iniciadas após assinatura dos Termos de Consentimento Livre e Esclarecido. O principal benefício direto do estudo para os participantes foi o fornecimento dos resultados dos exames laboratoriais. Quando constatados resultados fora dos parâmetros clínicos desejáveis foram anexadas cartas de orientações aos participantes e guia de encaminhamento para atendimento médico nas UBS, conforme previamente acordado com os gestores da Secretaria Municipal de Saúde.

\section{Resultados}

Da amostra populacional de 1339 indivíduos selecionados, $88,1 \%$ foram entrevistados, configurando-se uma perda de 159 (11,9\%), sendo $7,0 \%$ por recusa e $4,9 \%$ não encontrados em pelo menos três tentativas em horários e datas alternadas. Dos 1180 entrevistados, 967 (81,9\%) tiveram amostras sanguíneas coletadas e as mulheres foram a maioria $(55,4 \%)$.

A distribuição dos valores do perfil lipídico mostrou que $52,6 \%$ dos indivíduos que realizaram exames laboratoriais tinham CT igual ou superior a $200 \mathrm{mg} / \mathrm{dl}, 16,6 \% \mathrm{LDL}-\mathrm{C} \geq 160 \mathrm{mg} /$ dl, 34,4\% TG $\geq 150 \mathrm{mg} / \mathrm{dl}$ e $49,1 \%$ com a fração HDL-C não desejável $(<40 \mathrm{mg} / \mathrm{dl}$ em homens e $<$ 50 em mulheres) (Tabela 1$)$.

A prevalência de dislipidemias encontrada foi de $69,2 \%$, maior nas mulheres do que nos homens (RP de 1,10: IC 95\% 1,01-1,20), não sendo verificadas diferenças entre as faixas etárias ( $\mathrm{p}=$ 0,442) (Tabela 2). Entre os 669 indivíduos com dislipidemias, $35,0 \%(\mathrm{n}=234)$ relataram que sabiam da condição. Entre estes, 41,0\% (n = 108) faziam tratamento medicamentoso. Entre os que não reconheciam as alterações dos lipídeos, 2,8\% utilizavam medicamentos hipolipemiantes. 
Tabela 1. Distribuição dos indivíduos segundo categorias das frações lipídicas e sexo. Projeto VIGICARDIO, Cambé (PR), 2011.

\begin{tabular}{|c|c|c|c|c|c|c|c|c|}
\hline \multirow{2}{*}{ Lípides } & \multirow{2}{*}{$\operatorname{VR}(\mathrm{mg} / \mathrm{dl})$} & \multirow{2}{*}{ Categoria } & \multicolumn{2}{|c|}{ Mulheres } & \multicolumn{2}{|c|}{ Homens } & \multicolumn{2}{|c|}{ Total } \\
\hline & & & $\mathbf{n}$ & $\%$ & $\mathbf{n}$ & $\%$ & $\mathbf{n}$ & $\%$ \\
\hline \multirow[t]{3}{*}{ CT } & $<200$ & Desejável & 232 & 43,3 & 226 & 52,4 & 458 & 47,4 \\
\hline & 200 a 239 & Limítrofe & 196 & 36,6 & 133 & 30,9 & 329 & 34,0 \\
\hline & $\geq 240$ & Alto & 108 & 20,1 & 72 & 16,7 & 180 & 18,6 \\
\hline \multirow[t]{5}{*}{ LDL-C $^{*}$} & $<100$ & Ótimo & 93 & 17,6 & 79 & 19,4 & 172 & 18,4 \\
\hline & 100 a 129 & Desejável & 161 & 30,4 & 158 & 38,9 & 319 & 34,1 \\
\hline & 130 a 159 & Limítrofe & 179 & 33,8 & 110 & 27,0 & 289 & 30,9 \\
\hline & 160 a 189 & Alto & 74 & 14,0 & 47 & 11,5 & 121 & 12,9 \\
\hline & $\geq 190$ & Muito alto & 22 & 4,2 & 13 & 3,2 & 35 & 3,7 \\
\hline \multirow[t]{4}{*}{ HDL-C } & $>60$ & Desejável $^{\dagger}$ & 98 & 18,2 & 66 & 15,3 & 164 & 17,0 \\
\hline & 50 a 59 & Desejável $^{\dagger}$ & 144 & 26,9 & 58 & 13,5 & 202 & 20,9 \\
\hline & 40 a 49 & Desejável $^{\ddagger}$ & 172 & 32,1 & 126 & 29,2 & 298 & 30,8 \\
\hline & $<40$ & Baixo & 122 & 22,8 & 181 & 42,0 & 303 & 31,3 \\
\hline \multirow[t]{4}{*}{ TG } & $<150$ & Desejável & 364 & 67,8 & 271 & 62,9 & 635 & 65,6 \\
\hline & 150 a 199 & Limítrofe & 92 & 17,2 & 46 & 10,7 & 138 & 14,3 \\
\hline & 200 a 499 & Alto & 77 & 14,4 & 98 & 22,7 & 175 & 18,1 \\
\hline & $\geq 500$ & Muito alto & 3 & 0,6 & 16 & 3,7 & 19 & 2,0 \\
\hline
\end{tabular}

CT (colesterol total); LDL-C (lipoproteína de baixa densidade); HDL-C (lipoproteína de alta densidade); TG (triglicérides); VR = valores de referência; *31 amostras de material biológico não foram viáveis para realização da dosagem da fração; ${ }^{\dagger}$ Desejável para homens e mulheres; ${ }^{\ddagger}$ Desejável para homens.

Tabela 2. Prevalência de dislipidemias segundo sexo e faixa etária. Projeto VIGICARDIO-Cambé (PR), 2011.

\begin{tabular}{|c|c|c|c|c|c|c|}
\hline \multirow{3}{*}{$\begin{array}{l}\text { Faixa } \\
\text { etária }\end{array}$} & \multicolumn{4}{|c|}{ Tratamento } & & \\
\hline & \multicolumn{2}{|c|}{ Feminino $^{*}$} & \multicolumn{2}{|c|}{ Masculino $^{\dagger}$} & \multicolumn{2}{|c|}{ Total $^{\ddagger}$} \\
\hline & $\mathbf{n}$ & $\%$ & $\mathbf{n}$ & $\%$ & $\mathbf{n}$ & $\%$ \\
\hline 40-49 anos & 143 & 66,8 & 124 & 70,5 & 267 & 68,5 \\
\hline 50-59 anos & 126 & 75,0 & 86 & 63,7 & 212 & 70,0 \\
\hline 60-69 anos & 79 & 78,2 & 59 & 65,6 & 138 & 72,3 \\
\hline$>70$ anos & 38 & 71,7 & 14 & 46,7 & 52 & 62,7 \\
\hline Total & 386 & 72,0 & 283 & 65,7 & 669 & 69,2 \\
\hline
\end{tabular}

$\mathrm{p}=0,136 ;{ }^{\dagger} \mathrm{p}=0,077 ;{ }^{\ddagger} \mathrm{p}=0,442$

A prevalência de tratamento medicamentoso hipolipemiante entre os indivíduos com dislipidemias foi de $16,1 \%$, sem diferenças entre os sexos ( $p=0,188$ ) e com as frequências significativamente mais elevadas em idades mais avançadas $(\mathrm{p}<0,001)$. Entre os 108 indivíduos que faziam uso de medicamentos hipolipemiantes, 22,2\% (n =24) apresentaram perfil lipídico adequado, sem diferença significativa entre os sexos $(\mathrm{p}=0,935)$.

Dos indivíduos com dislipidemias em tratamento, $88,9 \%$ faziam uso de medicamentos do subgrupo farmacológico das estatinas, $13,9 \%$ do subgrupo dos fibratos e 1,9\% dos inibidores de absorção do colesterol. Entre as Estatinas, 81,5\% faziam uso da sinvastatina, $0,9 \%$ da pravastatina, $1,9 \%$ da fluvastatina, $2,8 \%$ da atorvastatina e $4,6 \%$ da rosuvastatina. A ezetimiba foi o único inibidor da absorção do colesterol utilizado por $1,9 \%$ dos entrevistados com dislipidemia. Quanto aos fibratos, $6,5 \%$ utilizavam bezafibrato, $4,6 \%$ ciprofibrato e $2,8 \%$ fenofibrato.

Verificou-se uma média de 1,04 fármacos hipolipemiantes em uso por indivíduo e 93,4\% faziam uso de apenas um. Em um caso verificou-se a combinação de fármacos (sinvastatina e ezetimiba).

Os medicamentos hipolipemiantes foram obtidos principalmente em farmácias e drogarias privadas $(52,2 \%)$, seguido pelos serviços de saúde pública $(33,6 \%)$ e os estabelecimentos privados vinculados ao Programa Aqui tem Farmácia Popular (11,5\%). Nos estabelecimentos próprios do SUS foram obtidos exclusivamente medicamentos do subgrupo farmacológico das estatinas, enquanto os inibidores da absorção do colesterol e os fibratos foram adquiridos nas drogarias e farmácias comerciais privadas (Tabela 3 ).

\section{Discussão}

A prevalência de dislipidemias de 69,2\%, determinada pela ausência de níveis ótimo ou desejável de qualquer das frações lipídicas e/ou pelo uso de 
Tabela 3. Locais de obtenção de medicamentos hipolipemiantes, por subgrupos farmacológicos e fármacos. Projeto VIGICARDIO, Cambé/PR, Brasil, 2011.

\begin{tabular}{|c|c|c|c|c|c|}
\hline \multirow{2}{*}{$\begin{array}{c}\text { Subgrupos farmacológicos } \\
\text { e fármacos }\end{array}$} & \multirow[b]{2}{*}{$\mathbf{n}$} & \multicolumn{4}{|c|}{ Locais de obtenção* $(\%)$} \\
\hline & & SUS & AFP & DFP & Plano \\
\hline Estatinas & 96 & 39,6 & 13,5 & 43,8 & 3,1 \\
\hline Sinvastatina & 88 & 42 & 14,8 & 39,8 & 3,4 \\
\hline Pravastatina & 1 & - & - & 100 & - \\
\hline Fluvastatina & 2 & - & - & 100 & - \\
\hline Atorvastatina & 3 & 33,3 & - & 66,7 & - \\
\hline Rosuvastatina & 5 & - & - & 100 & - \\
\hline Inibidores absorção do colesterol & 2 & - & - & 100 & - \\
\hline Ezetimiba & 2 & - & - & 100 & - \\
\hline Fibratos & 15 & - & - & 100 & - \\
\hline Bezafribato & 7 & - & - & 100 & - \\
\hline Ciprofibrato & 5 & - & - & 100 & - \\
\hline Fenofibrato & 3 & - & - & 100 & - \\
\hline Total & & 33,6 & 11,5 & 52,2 & 2,7 \\
\hline
\end{tabular}

*SUS: Sistema Único de Saúde; AFP: Farmácias e drogarias privadas conveniadas ao Programa Aqui tem Farmácia Popular; DFP: Drogarias e farmácias privadas com pagamento direto pelo usuário: Plano: Planos de saúde ou similares; Outros: Entidades filantrópicas ou consultórios médicos.

medicamentos hipolipemiantes, estabeleceu uma situação preocupante. Clinicamente, as altas prevalências de níveis séricos indesejáveis de triglicerídeos e de HDL-C não podem ser negligenciadas, considerando seu importante papel no processo aterogênico, no qual os triglicerídeos exercem ação indutora no aumento de fibrinogênio e nos fatores da coagulação, e o HDL-C por não desenvolver seu papel antiteratogênico e oposto ao do LDL-C, quando em concentrações baixas ${ }^{14}$.

Em comparação com o estudo de base populacional realizado em Campos de Goytacazes/RJ ${ }^{15}$, com indivíduos a partir de 18 anos, as prevalências das frações lipídicas estimadas no presente estudo foram bem mais elevadas. Análise realizada por Pozzan et al. ${ }^{16}$ com indivíduos de 20 a 39 anos mostrou similaridade com os resultados presentes. No município de Ribeirão Preto, com indivíduos a partir de 30 anos, verificou-se prevalência bastante próxima $(61,9 \%)$, com alterações das frações lipídicas oscilando de $20,4 \%$ a $43,7 \%{ }^{17}$.

Estudo realizado em Portugal, de base populacional, verificou aumento das taxas de hipercolesterolemia com a progressão da idade, que variou de $47 \%$ em menores de 40 anos a $64 \%$ entre aqueles de 60 e 74 anos, ao mesmo tempo em que a prevalência de hipertrigliceridemia aumentava de $47 \%$ para $59 \%$ nas referidas faixas etárias ${ }^{18}$. Em investigação realizada no Estado americano de New England (USA), com registros efetuados por médicos de família, verificou-se prevalência de dislipidemias de 52,3\% entre adultos ${ }^{19}$. É provável que parte das diferenças relatadas, com maior prevalência no nosso estudo, deva-se à inclusão de indivíduos a partir de 40 anos, pois se observou aumento contínuo da prevalência de dislipidemias nas faixas etárias mais avançadas. A redução da prevalência a partir de 70 anos, nos homens, pode ser sugestiva de óbitos masculinos precoces e inversão do perfil epidemiológico das mortes por doenças cardiovasculares. As maiores médias dos níveis séricos de CT e LDL-C encontradas nas mulheres e de TG nos homens, também foram mostradas em outros estudos realizados no país ${ }^{15,16}$, revelando distintos perfis em cada sexo.

Outros estudos, a partir de dados autorreferidos como em oito cidades do Estado de São Paulo $^{20}$, ou envolvendo moradores de capitais de estados brasileiros entrevistados por via telefônica, no âmbito do Sistema VIGITEL ${ }^{21}$, e com participantes de um evento de educação em saúde realizado no interior do Estado de São Paulo ${ }^{22}$, identificaram prevalências bem menores de dislipidemias $(12,2 \%, 15,8 \%$ e $25,8 \%$, respectivamente). Estes resultados estão de acordo com o baixo reconhecimento das dislipidemias verificado na presente investigação, pois apenas um terço dos indivíduos com alterações das frações lipídicas relatou reconhecer a presença da condição.

A prevalência de tratamento medicamentoso foi de apenas $16,1 \%$, porém não foram encontrados estudos que avaliassem a necessidade de farmacoterapia, o que dificultou a comparação dos 
resultados. Sabe-se que para o controle das dislipidemias, as terapias nutricionais e as mudanças de estilo de vida são sempre recomendadas à priori, e, a critério clínico, deve-se instituir o tratamento medicamentoso ${ }^{5}$ quando mudanças nos hábitos não são suficientes para induzir uma diminuição efetiva do risco cardiovascular ${ }^{23}$.

A maior prevalência de tratamento medicamentoso em idosos no estudo pode ter sido influenciada pela maior utilização dos serviços de saúde pelos usuários desse grupo etário, pois a associação de uso de serviços com o aumento da idade tem sido descrita ${ }^{24}$.

A constatação de que $2,8 \%$ dos indivíduos com dislipidemias que não reconheciam a condição encontravam-se em tratamento medicamentoso sugere problemas na atenção, como desinformação e ausência de aconselhamento profissional. Por outro lado, do ponto de vista farmacoterapêutico, a disponibilização de medicamentos por si só não garante que as metas terapêuticas para o controle dos lipídeos sejam atingidas.

Sobre o uso de medicamentos, três aspectos merecem ser destacados: a realização de tratamento predominantemente em regime de monoterapia $(93,4 \%)$, uso expressivo de medicamentos com monofármacos e utilização de estatinas $(85, \%)$ muito superior à dos demais subgrupos farmacológicos dos hipolipemiantes. O predomínio da monoterapia para o controle dos lipídeos também foi constatado por estudo realizado no estado de Santa Catarina $(98,3 \%)$, sendo este regime terapêutico referido como um dos princípios de simplificação da farmacoterapia que melhora a adesão ao tratamento ${ }^{25}$. Com relação ao uso das estatinas, o Protocolo Clínico e as Diretrizes Terapêuticas do tratamento das dislipidemias no Programa de Medicamentos Excepcionais alerta para o risco de ocorrência de casos de rabdomiólise, mioglobinúria e necrose tubular aguda associados. Estes eventos são raramente relatados em regime de monoterapia, associando-se principalmente ao uso concomitante com outros fármacos (fibratos, niacina, ciclosporina) e indivíduos com múltiplas comorbidades (idosos, portadores de insuficiência renal, etc.) ${ }^{6}$.

A associação das estatinas com a ezetimiba é recomendada nos casos em que as metas terapêuticas de redução dos lipídeos não são atingidas, devendo ser utilizada criteriosamente, pois, embora seus efeitos colaterais sejam raros, há risco de ocorrência de rabdomiólise ${ }^{5}$. Com relação aos fibratos, sabe-se que podem ser úteis na prevenção de doenças microvasculares na presença de diabetes do tipo 2, de forma isolada ou em as- sociação com estatinas, embora não se tenha resultados consistentes de estudos clínicos que demonstrem o benefício de seu uso em monoterapia na redução de eventos cardiovasculares ${ }^{5}$.

Schul $^{23}$ destaca que, apesar da monoterapia modificar os níveis de lípides, a combinação de drogas pode ser necessária para um manuseio mais abrangente, principalmente nas situações de alto risco cardiovascular, no qual se exige reduções mais acentuadas, com estatinas mais potentes ou em associação com drogas que diminuam a absorção de colesterol.

A predominância de uso das estatinas, possivelmente, deva-se à sua inclusão nos programas de Assistência Farmacêutica, a partir de 2002, na lista de medicamentos fornecidos no Programa de Medicamentos Excepcionais, como opção terapêutica para o controle das dislipidemias em pacientes com alto risco cardiovascular, sendo padronizados os fármacos sinvastatina, atorvastatina e pravastatina ${ }^{6}$. Esta lista foi ampliada em 2006 com a inclusão da fluvastatina e de fármacos do subgrupo químico fibratos: bezafibrato, ciprofibrato, etofibrato, fenofibrato e genzibrozila ${ }^{7}$.

Outras modificações nas condições de acesso à sinvastatina ocorreram em 2009, com destaque para a transferência da sinvastatina para o Componente Básico da Assistência Farmacêutica ${ }^{8}$ e o estabelecimento da responsabilidade de financiamento da sinvastatina aos municípios, estados e/ou união, conforme pactuação em Comissões Intergestores Bipartite ${ }^{26}$. Estas duas alterações normativas definiram a municipalização da distribuição e o compartilhamento do custeio entre as gestões estaduais e municipais. Neste intervalo de tempo, entretanto, o Governo Federal já havia autorizado a Fundação Oswaldo Cruz a disponibilizar medicamentos, mediante ressarcimento, à população atendida pelo SUS, dando início à implantação do Programa Farmácia Popular do Brasil (PFP), com a Lei 10.858 de 13/04/2004, que foi regulamentada na sequência pelo Decreto 5.090 de $20 / 05 / 2004$, que previa a possibilidade de participação subsidiada da rede privada no programa ${ }^{27}$. Inicialmente, os medicamentos elencados destinavam-se a garantir o acesso da população a medicamentos para tratamento da HA e DM, sendo as sinvastatinas incluídas apenas em $2010^{9}$.

Embora estas políticas tivessem o objetivo de garantir acesso universal ao tratamento medicamentoso das dislipidemias, com ênfase na participação do setor público como seu fornecedor, neste estudo foi observado que as farmácias e as drogarias privadas continuaram a ser o principal local de obtenção dos hipolipemiantes, seguidas 
pelas unidades assistenciais do serviço municipal de saúde e pelos estabelecimentos privados credenciados pelo PAFP. Ressalte-se que os estabelecimentos vinculados aos serviços municipais forneceram unicamente a sinvastatina, sendo que os fibratos e os inibidores de absorção de colesterol foram adquiridos mediante pagamento direto pelos usuários.

Com relação ao bezafibrato, que tem indicação clínica em casos de hipertrigliceridemia em portadores de $\mathrm{DM}^{23}$, há que se considerar a hipótese de que ocorra limitação no tratamento medicamentoso das dislipidemias devido à sua indisponibilidade nos serviços públicos.

O controle das dislipidemias dos indivíduos que estavam em tratamento medicamentoso no presente estudo foi muito baixo $(22,2 \%)$, principalmente se comparado com o resultado de uma pesquisa com pessoas com hipertensão $(55,38 \%)^{28}$. Pode ser que a diferença entre as taxas de controle nos estudos seja decorrente da melhor adesão farmacoterapêutica na presença de comorbidades. Porém, para os autores, a situação de baixo controle - ainda que bastante superior ao verificado em nosso estudo - indica a necessidade de adoção de terapias mais agressivas, maior persuasão para a aderência dos pacientes ao tratamento e melhorias nas políticas de saúde pública.

Do ponto de vista do acesso aos fármacos hipolipemiantes, a menor participação do setor público observada no presente estudo pode indicar restrição da oferta e sugere possível insuficiência das políticas públicas para assistência farmacêutica das dislipidemias. Assim, é possível que a baixa prevalência de tratamento medicamentoso e de controle dos lipídeos séricos encontrada decorra de diagnósticos tardios das dislipidemias e/ou insuficiente disponibilidade de medicamentos para tratamento. Mesmo reconhecendo-se que o controle das dislipidemias pode ser obtido por medidas terapêuticas não medicamentosas, sendo moderado por fatores individuais envolvidos no processo, o uso dos medicamentos, a acessibilidade e a adequada gestão clínica do processo farmacoterapêutico também são aspectos relevantes.

De modo que podem ter contribuído para o baixo controle das dislipidemias o não reconhecimento da condição pelos indivíduos, a inadequada gestão clínica das doenças - que inclui a obtenção de bons níveis de adesão terapêutica dos pacientes - e/ou as dificuldades pontuais de implementação das políticas públicas de assistência farmacêutica.

Por último, não se pode desconsiderar as limitações do estudo, tais como a realização de uma única medida laboratorial, acrescidos dos possíveis erros não controlados como a não realização do jejum no período recomendado, e a identificação de interferências de fatores individuais ou circunstanciais, como a presença de doenças agudas, a atividade física intensa ou as características das dietas adotadas nos dias que antecederam a coleta.

Como pontos positivos deste estudo destacam-se a realização de exames laboratoriais em estudo de base populacional com elevada taxa de resposta, a averiguação de todos os medicamentos utilizados e a semelhança da distribuição etária e por sexo entre a amostra da totalidade dos entrevistados e da coleta de exames laboratoriais, sugerindo representatividade das características demográficas da população total do município.

Conclui-se que a elevada prevalência de dislipidemias na população estudada, a baixa frequência de reconhecimento da condição e de controle pelos indivíduos em tratamento medicamentoso reforçam a relevância clínica e epidemiológica das dislipidemias na população adulta e idosa. Do ponto de vista da gestão dos medicamentos, o fato de as farmácias e drogarias privadas constituírem os principais locais de obtenção de hipolipemiantes, mediante aquisição com pagamento direto, sugere alcance limitado das políticas públicas de controle das dislipidemias, seja pela inadequação da oferta ou ainda por desconhecimento dos usuários do sistema público sobre a disponibilidade destes medicamentos.

\section{Colaboradores}

AJ Petris trabalhou na concepção, na pesquisa e na metodologia, RKT de Sousa contribuiu na concepção e em todas as fases de construção do artigo e MSS Bortoleto esteve envolvida na pesquisa, na metodologia e na análise de dados. Todos os autores participaram da revisão final do trabalho.

\section{Agradecimentos}

Pelo financiamento parcial do edital pró-Equipamentos (Convênio Capes/Pró-Equipamento) e pelos recursos do Programa de Apoio a Pós-Graduação (PROAP/CAPES). 


\section{Referências}

1. Bonow RO. Prevention of cardiovascular disease: challenges and opportunities. Ital Heart J 2004; 5(Supl. 6):6S-8S.

2. Tunstall-Pedoe H. Preventing Chronic Diseases. A Vital Investment. World Health Organization Global Report 2006; 35(4):1107-1107.

3. Kesteloot H, Sans S, Kromhout D. Dynamics of cardiovascular and all-cause mortality in Western and Eastern Europe between 1970 and 2000. Eur Heart J 2006; 27(1):107-113.

4. Brasil. Ministério da Saúde (MS). Prevenção clínica de doenças cardiovasculares, cerebrovasculares e renais. Brasília: MS; 2006.

5. Xavier H, Izar M, Faria Neto J, Assad M, Rocha V Sposito A, Fonseca FA, Santos JE, Santos RD, Bertolami MC, Faludi AA, Martinez TLR, Diament J, Guimarães A, Forti NA, Moriguchi E, Chagas ACP, Coelho OR, Ramires JAF. V Diretriz brasileira de dislipidemias e Prevenção da aterosclerose. Arq Bras Cardiol 2013; 101(4):1-20.

6. Brasil. Ministério da Saúde (MS). Secretaria de Assistência. Departamento de Sistemas e redes Assistênciais. Protocolos clinicos e diretrizes terapêuticas: medicamentos excepcionais. Brasília: MS; 2002.

7. Brasil. Portaria n².577/06, de 27 de outubro de 2006. Aprova o componente de medicamentos de dispensação excepcional. Diário Oficial da União 2006; 27 out.

8. Brasil. Portaria $\mathrm{n}^{\circ} 2.981 / 09$, de 26 de novembro de 2009. Aprova o componente especializado da assistência farmacêutica. Diário Oficial da União 2009; 26 nov.

9. Brasil. Portaria $n^{\circ} 184$, de 3 de fevereiro de 2011. Dispõe sobre o Programa Farmácia Popular do Brasil. Diário Oficial da União 2011; 3 fev.

10. Instituto Brasileiro de Geografia e Estatística (IBGE). Cidades. Rio de Janeiro: IBGE; 2013.

11. Cambé. Secretaria Municipal de Saúde de Cambé (SMSC). Plano municipal de saúde 2010-2011. Cambé: SMSC; 2010.

12. Paniz VM, Fassa AG, Facchini LA, Piccini RX, Tomasi E, Thume E, Silveira DS, Rodrigues MA, Domingues MR, Dâmaso AB. Free access to hypertension and diabetes medicines among the elderly: a reality yet to be constructed. Cad Saude Publica 2010; 26(6):1163-1174.

13. World Health Organization (WHO). Collaborating Centre for Drug Statistics Methodology. International language for drug utilization research ATC/DDD. Geneva: WHO; 2013.

14. Juliá V JN, Ramos VC. Dislipemia y aterosclerosis: patogenia y fisiopatología. In: Puig JG, Hernández PLM. Conceptos actuales en dislipemia y riesgo vascular. Madri: Farmalia Comunicación; 2009. p. 51-66.

15. Souza LJ, Souto Filho JTD, de Souza TF, Reis AFF, Neto CG, Bastos DA, Côrtes VA, Chalita FEB, Teixeira CL. Prevalência de dislipidemia e fatores de risco em Campos dos Goytacazes-RJ. Arq. bras. cardiol 2003; 81(3):249-256

16. Pozzan R, Brandão AA, Magalhães MEC, de Souza NA, Silva APB. Níveis lipídicos em uma série de casos da cidade do Rio de Janeiro. Revista da SOCERJ 2005; 18(6):547-558
17. Moraes SA, Checchio MV, Freitas IC. Dyslipidemia and correlates in adults living in Ribeirao Preto, SP: results of the EPIDCV Project. Arq Bras Endocrinol Metabol 2013; 57(9):691-701.

18. Perdigão C, Duarte J, Santos A. Prevalência e caracterização da Hipercolesterolemia em Portugal. Implicações numa estratégia de Prevenção. Uma análise do Estudo AMALIA. Factores de Risco 2009; 17:12-19.

19. Tabenkin H, Eaton CB, Roberts MB, Parker DR, McMurray JH, Borkan J. Differences in cardiovascular disease risk factor management in primary care by sex of physician and patient. Ann Fam Med 2010; 8(1):25-32.

20. Fernandes RA, Christofaro DG, Casonatto J, Codogno IS, Rodrigues EQ, Cardoso ML, Kawaguti SS, Zanesco A. Prevalence of dyslipidemia in individuals physically active during childhood, adolescence and adult age. Arq Bras Cardiol 2011; 97(4):317-323.

21. Sá NN, Moura EC. Factors associated with the burden of metabolic syndrome diseases among Brazilian adults. Cad Saude Publica 2010; 26(9):1853-1862.

22. Coltro RS, Mizutani BM, Mutti A, Delia MP, Martinelli LM, Cogni AL, Matsubara BB. Cardiovascular risk factors in a population attending a community event on health education. Rev Assoc Med Bras 2009; 55(5):606610.

23. Schulz I. Treatment of dyslipidemia: how and when to combine lipid lowering drugs. Arq Bras Endocrinol Metabol 2006; 50(2):344-359.

24. Paskulin LM, Valer DB, Vianna LA. Use and access of the elderly to primary health care services in Porto Alegre (RS, Brasil). Cien Saude Colet 2011; 16(6):29352944.

25. Cunico C. Dislipidemia e efetividade do uso de hipolipemiantes em população do extremo oeste do Estado de Santa Catarina [dissertação]. Curitiba: Universidade Federal do Paraná; 2013.

26. Brasil. Portaria n4.217, de 28 de dezembro de 2010. Aprova as normas de financiamento e execução do componente básico da assistência farmacêutica. Diário Oficial da União 2010; $28 \mathrm{dez}$.

27. Brasil. Lei $n^{\circ} 10.858$, de 13 de abril de 2004. Autoriza a Fundação Oswaldo Cruz- Fiocruz a disponibilizar medicamentos mediante ressarcimento, e dá outras providências. Diário Oficial da União 2004; 13 abr.

28. Três GS, Utzig JB, Martins R, Heinrich S. Controle da pressão arterial, do diabetes mellitus e da dislipidemia na população de hipertensos de um ambulatório de residência médica. Rev Bras Hipertens 2009; 16(3):143147.

Artigo apresentado em 15/05/2015

Aprovado em 03/09/2015

Versão final apresentado em 05/09/2015 